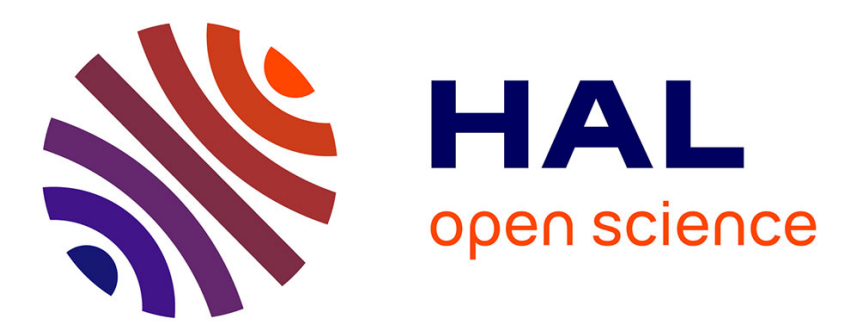

\title{
Continuous synthesis of high quality CdSe quantum dots in supercritical fluids
}

Arkajyoti Chakrabarty, Samuel Marre, Ryan F. Landis, Vincent M. Rotello, Uday Maitra, André Del Guerzo, Cyril Aymonier

\section{To cite this version:}

Arkajyoti Chakrabarty, Samuel Marre, Ryan F. Landis, Vincent M. Rotello, Uday Maitra, et al.. Continuous synthesis of high quality CdSe quantum dots in supercritical fluids. Journal of Materials Chemistry C, 2015, 3 (29), pp.7561-7566. 10.1039/c5tc01115a . hal-01179462

\section{HAL Id: hal-01179462 \\ https://hal.science/hal-01179462}

Submitted on 3 Jun 2021

HAL is a multi-disciplinary open access archive for the deposit and dissemination of scientific research documents, whether they are published or not. The documents may come from teaching and research institutions in France or abroad, or from public or private research centers.
L'archive ouverte pluridisciplinaire HAL, est destinée au dépôt et à la diffusion de documents scientifiques de niveau recherche, publiés ou non, émanant des établissements d'enseignement et de recherche français ou étrangers, des laboratoires publics ou privés. 


\title{
Continuous synthesis of high quality CdSe quantum dots in supercritical fluids
}

Arkajyoti Chakrabarty ${ }^{\text {ab }}$, Samuel Marre ${ }^{a}$, Ryan F. Landis ${ }^{c}$, Vincent M. Rotello ${ }^{c}$, Uday Maitra ${ }^{d}$, André Del Guerzo ${ }^{b}$ and Cyril Aymonier *a

${ }^{a}$ CNRS, Univ. Bordeaux, ICMCB, UPR9048, F-33600 Pessac, France / E-mail: cyril.aymonier@icmcb.cnrs.fr bUniv. Bordeaux, CNRS, Institut des Sciences Moléculaires, UMR5255, 351 Cours de la Libération, 33400 Talence, France 'Department of Chemistry, University of Massachusetts Amherst, 710 North Pleasant Street, Amherst, MA 01003, USA

${ }^{\mathrm{d} D e p a r t m e n t}$ of Organic Chemistry, Indian Institute of Science, Bangalore 560012, India

\begin{abstract}
:
We demonstrate in here a powerful scalable technology to synthesize continuously high quality CdSe quantum dots (QDs) in supercritical hexane. Using a low cost, highly thermally stable Cd-precursor, cadmium deoxycholate, the continuous synthesis is performed in $400 \mu \mathrm{m}$ ID stainless steel capillaries resulting in CdSe QDs having sharp full-width-at-half-maxima $(23 \mathrm{~nm})$ and high photoluminescence quantum yields (45-55\%). Transmission electron microscopy images show narrow particles sizes distribution $(\sigma \leq 5 \%)$ with well-defined crystal lattices. Using two different synthesis temperatures $\left(250^{\circ} \mathrm{C}\right.$ and $310^{\circ} \mathrm{C}$ ), it was possible to obtain zinc blende and würtzite crystal structures of CdSe QDs, respectively. This synthetic approach allows achieving substantial production rates up to $200 \mathrm{mg}$ of QDs per hour depending on the targeted size, and could be easily scaled to gram per hour.
\end{abstract}

Note : † Electronic supplementary information (ESI) available: Calculation of quantum yields of CdSe QDs at different excitation wavelengths based on the excitation, absorption spectra and experimental quantum yields, example of calculation of production rate. See DOI: 10.1039/c5tc01115a

\section{Introduction}

The size-dependent optical and electronic properties ${ }^{1}$ of II-VI semiconductor nanocrystal (NC) quantum dots (QDs) such as CdS, CdSe or CdTe, make them key materials for application in optoelectronics, ${ }^{2}$ photovoltaics, ${ }^{3}$ biomedical imaging, biological labelling ${ }^{4}$ and LEDs. ${ }^{5}$ In the latter, CdSe based QD-LEDs should provide suitable alternatives to the OLEDs in the coming years due to their easy solution-processability and pure color, low power consumption, low-cost manufacture and ultrathin, transparent, flexible form factors. ${ }^{6}$

However, these applications require narrow and symmetric emission line-shapes and high photoluminescence quantum yields, necessitating high particle uniformity and crystallinity over wide range of particle sizes. Achieving these properties in a scalable fashion requires the development of powerful and efficient synthetic techniques. 
Although there are numerous batch mode methods in the literature for the synthesis of CdSe QDs, ${ }^{7}$ only few report on the synthesis of CdSe QDs at microscale in a continuous manner. Most of the examples so far have reported the use of high boiling point liquid solvents. Two main strategies are considered: (i) the single phase flow approaches in liquid phase ${ }^{8}$ or (ii) the segmented flow approaches in slugs or droplets using either gasliquid $^{9}$ or liquid-liquid ${ }^{10}$ microflows. The latter cases ensured a good control over the residence time distribution and thus the final size distribution of the synthesized QDs, although these approaches are limited to conditions for which both the continuous phase and the reactive phase are non-miscible. Continuous micro-flow synthesis of nanoparticles such as QDs has several advantages over the conventional batch modes due to enhancement of mass and heat transfer, ${ }^{11}$ precise screening of reaction parameters, low reagent consumption during optimization process and very good reproducibility. ${ }^{12}$

By performing the reactions at high pressures, it is possible to attain regimes where the solvents and the reagents are either liquid or supercritical. These allow expanding the operating regimes for nanomaterial syntheses. In the supercritical regime, the fluids behave both as liquids and gases, as the major advantages being low viscosities (i.e. high miscibility and faster diffusion rates) and sufficiently high densities for dissolving a wide range of compounds with low to medium polarity..$^{13}$ These lead to narrow residence time distributions (RTD), which in turn help accessing narrow size distributed nanoparticles. ${ }^{14}$ Although Marre et al. performed the pioneering work on the continuous "on chip" synthesis of narrow size distribution of CdSe quantum dots in supercritical hexane, ${ }^{14 a}$ the small volume of the microreactor strongly limited the production rate.

In this work, we report a simple, low-cost technique to prepare CdSe quantum dots in large scale with excellent optical properties and narrow size distribution. The reactions were carried out at high pressure (10 MPa) and temperatures $\left(250^{\circ} \mathrm{C}<T<310^{\circ} \mathrm{C}\right)$ inside standard stainless-steel capillaries in supercritical hexane, making the system both readily fabricated and scalable. Additionally, we used a simple approach for preparing precursor solutions using a highly thermally stable cadmium precursor, cadmium deoxycholate $\left(\mathrm{Cd}(\mathrm{DCh})_{2}\right)$, as the cadmium source. ${ }^{15}$ The bile acid based Cd-precursor has the advantage over other commonly used Cdprecursors (e.g. Cd-oleate, myristate, styrate, etc.) as it is much less expensive and can easily be prepared in a one-pot synthesis (decomposition temperature of $\mathrm{Cd}(\mathrm{DCh})_{2}: 332^{\circ} \mathrm{C}$ ).

\section{Experimental details}

\section{Chemicals}

Trioctylphosphine (TOP, assay 97\%), Se powder, cadmium nitrate tetrahydrate, sodium deoxycholate, oleylamine (OAm, technical grade, 70\%) were purchased from Sigma-Aldrich and Fluka. Methanol, chloroform, acetone, ethanol, anhydrous hexane were purchased from Sigma-Aldrich. Fluorescence reference standard dyes coumarin 153 and rhodamine 101 were purchased from Sigma-Aldrich and Exciton.

\section{Preparation of precursor solutions}

A previously reported procedure was adopted to prepare the cadmium deoxycholate $\left(\mathrm{Cd}(\mathrm{DCh})_{2}\right)$ precursor in large scale. ${ }^{14}$ In this process, $\mathrm{Cd}\left(\mathrm{NO}_{3}\right)_{2} \cdot 4 \mathrm{H}_{2} \mathrm{O}$ and sodium deoxycholate were stirred in methanol at room temperature to obtain a white gelatinous precipitate, which was isolated by filtration and dried subsequently.

Hexane (critical coordinates: $T_{c}=234^{\circ} \mathrm{C}, P_{c}=3.01 \mathrm{MPa}$ ) was used as solvent for the continuous supercritical fluids synthesis of CdSe QDs. Cadmium deoxycholate $(0.25 \mathrm{mmol})$ was dissolved in oleylamine $(9.1 \mathrm{mmol})$ and trioctylphosphine $(4.5 \mathrm{mmol})$ upon heating at $80^{\circ} \mathrm{C}$ for $15 \mathrm{~min}$ and the resulting solution was cooled and diluted in hexane at room temperature. Trioctylphosphine selenide was prepared by dissolving selenium $(0.38 \mathrm{mmol})$ in TOP ( $2.3 \mathrm{mmol})$ upon heating at $80^{\circ} \mathrm{C}$ for $10 \mathrm{~min}$ and further dilution with hexane at room temperature. Both $\mathrm{Cd}$ and Se precursor solutions were carefully degassed with argon before running the microfluidic setup. The reaction temperature was fixed at either $250^{\circ} \mathrm{C}$ or $310^{\circ} \mathrm{C}$ to synthesize zinc blende (ZB) and würtzite (W) CdSe 
NCs, respectively. Flowrates were controlled by high pressure syringe pumps and the residence times were tuned from 5 to 60 seconds.

\section{Experimental set-up}

The reaction set-up was engineered to provide strict control over nucleation and growth of the CdSe NCs. The reactor consists of $1 / 32^{\prime \prime}$ stainless steel (ss) microtubing (length $=1 \mathrm{~m}$, internal diameter of $400 \mu \mathrm{m}$ ) coiled over a cylindrical heating cartridge connected to a Eurotherm temperature controller (Fig. 1). The microtubings were connected to high pressure syringe pumps (Harvard Apparatus, PHD 2000) with conventional Swagelok fittings. Three-way valves were used to control the fluid flow inside the microtubing. The $\mathrm{Cd}$ and Se solutions were separately introduced into the microreactor: the cadmium precursor was first preheated at the desired temperature $\left(250\right.$ or $310^{\circ} \mathrm{C}$ ) before being mixed with the room temperature selenium precursor to nucleate the CdSe QDs. Subsequently, the nucleated NCs underwent growth inside a second 1/32" ss tubing, with the residence time $\left(R_{t}\right)$ of the NCs tuned by adjusting the flowrate. A back pressure regulator (JASCO BP-2080) was used to maintain high pressure (10 $\mathrm{MPa}$ ) and to recover transparent QDs solutions at room temperature at the end of the continuous reaction sequence.

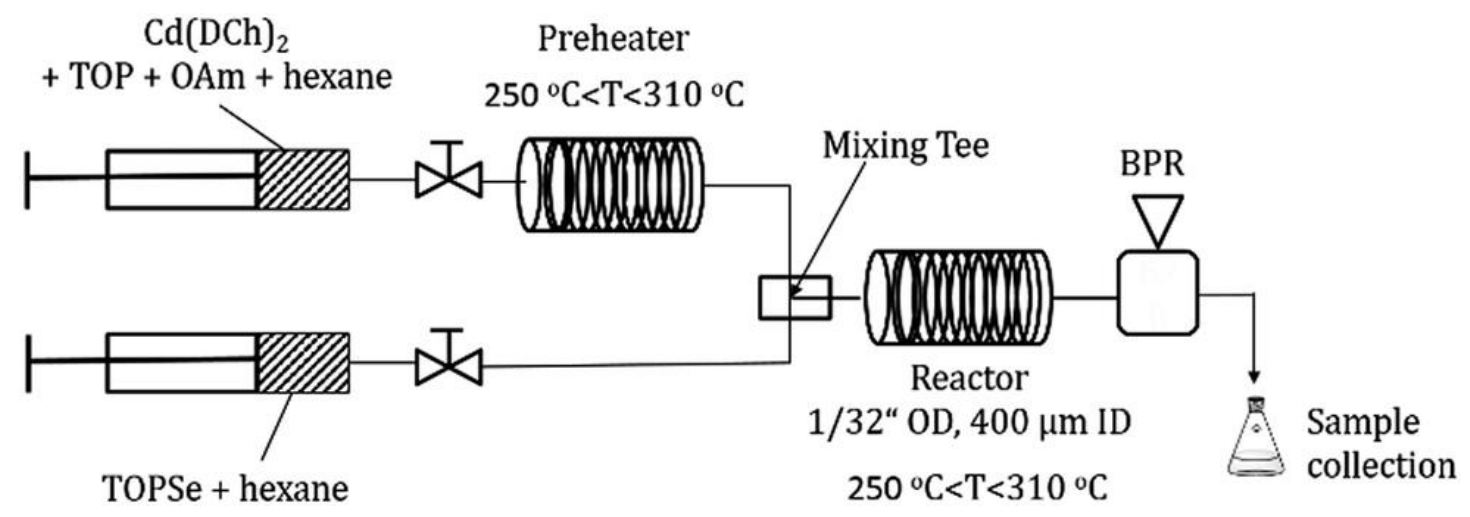

Figure 1 : Continuous supercritical set-up for the synthesis of high quality CdSe QDs.

CdSe QDs of different sizes were obtained depending on (i) the residence time and (ii) the temperature. The residence times $\left(R_{\mathrm{t}}\right)$ upon mixing were calculated according to the following equation:

$$
R_{\mathrm{t}}=\frac{V_{\text {reactor }}}{Q_{\text {tot }}} \times \frac{\rho_{\text {conditions }}}{\rho_{\text {syringe }}}
$$

where $V_{\text {reactor }}$ is the volume $\left(\mu \mathrm{m}^{3}\right)$ of the microreactor, $Q_{\text {tot }}\left(\mu \mathrm{m}^{3} \mathrm{~s}^{-1}\right)$ is the total flowrate of the syringe pumps, $\rho_{\text {conditions }}$ is the density of the fluid in the hot region (i.e. $P=10 \mathrm{MPa} ; 250^{\circ} \mathrm{C}<T<310^{\circ} \mathrm{C}$ ) and $\rho_{\text {syringe }}$ is the density of the fluid in the cold region (i.e. $P=10 \mathrm{MPa} ; T=\mathrm{RT}$ ).

\section{Characterization techniques}

The as-prepared CdSe QDs solutions (Fig. 2) were diluted with hexane or chloroform to prepare the solutions for absorption and photoluminescence $(\mathrm{PL})$ measurements. Optical absorption spectra were recorded using a Varian Cary 5000 UV-Vis NIR spectrophotometer and PL spectra were recorded using a Horiba Scientific Fluoromax-4 Spectrofluorometer. For PL quantum yield (QY) measurements, the absorbance of the CdSe QDs and the reference dye were adjusted to be comparable $(A<0.1)$. Photoluminescence of the same QDs and dye samples were recorded using the same excitation wavelengths, but due to the small Stokes shift in the NCs, the 
excitation had to be performed at $\lambda_{\mathrm{ex}}<\lambda_{\max }$ of absorption. The integrated area under the fluorescence curve was computed and the PL QY was calculated using standard equations. ${ }^{16}$

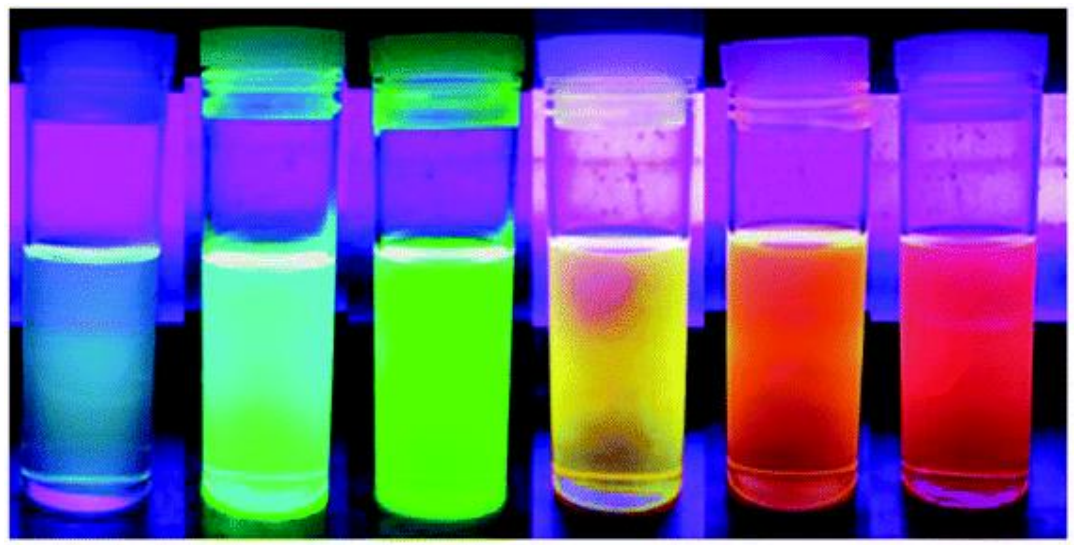

Figure 2 : Photographs of highly luminescent CdSe NCS of various sizes synthesized from cadmium deoxycholate precursor through the continuous mode in supercritical hexane (at $P=10 \mathrm{MPa} ; 250^{\circ} \mathrm{C}<T<310{ }^{\circ} \mathrm{C}$ ) showing emissions from blue to red regions of the visible spectrum (using UV-lamp at an excitation wavelength of $365 \mathrm{~nm}$ ).

For TEM characterization, the as-prepared QDs solutions were purified by precipitation using anhydrous acetone and centrifuged at $10000 \mathrm{rpm}$ to isolate the NCs. The process was repeated six times to ensure the removal of excess capping ligands. The purified CdSe QDs were then re-dispersed in hexane or chloroform and dilute solutions $(4-5 \mu \mathrm{L})$ of the samples were drop-casted over the carbon-coated copper grids and evaporated before the measurement. HRTEM images were recorded with a JEOL 2200 FS microscope, operating at $200 \mathrm{kV}$ with a field emission gun and point resolution of $0.23 \mathrm{~nm}$. Powder X-Ray diffraction measurements were obtained using a PANalytical X'Pert Pro Powder Diffractometer in the Brag-Brentano geometry using a monochromator with $\mathrm{K}_{1}$ line $(\lambda=1.54056 \AA)$ at room temperature with $2 \theta$ range $8-80^{\circ}$ and the CdSe QD solutions were drop-casted onto a silicon substrate.

\section{Results and discussion}

\section{Nucleation and growth of CdSe QDs inside the microtubing}

The CdSe particles sizes distribution mainly depends on residence time $\left(R_{\mathrm{t}}\right)$ and residence time distribution (RTD). Since flows are laminar within the microreactor (Reynold number ranges typically between 50 and 500 in our conditions), the velocity exhibits a parabolic profile leading to a spreading of the RTD through the dispersion mechanism. Supercritical fluids (SCFs) help narrowing this RTD thanks to their intrinsic properties of low viscosity and high diffusivity. ${ }^{17}$ In the supercritical microscale process, the high super-saturation provided by hexane results in a large number of nuclei due to complete depletion of the monomers. As a result, after the initial nucleation only time-controlled growth by coalescence is assumed without any further second nucleation. This conclusion is supported by the decrease of the CdSe concentration with residence time (Fig. 4c). The narrow RTD coupled with high super-saturation leads to a narrowing of the size distribution of CdSe QDs obtained using this continuous SCF strategy.

NCs with excellent optical properties were obtained at $T=250^{\circ} \mathrm{C}$ for the reaction yielding zinc blende (ZB) CdSe NCs. These NCs have absorption features where the first and second excitonic peaks were further apart. ${ }^{18}$ The PL and absorption spectra of the NCs for different residence times are described in Fig. 3 . The NCs showed multiple excitonic absorption peaks (4 features) and sharp PL peaks (full-width-at-half-maximum, FWHM = 23 $\mathrm{nm}$ ). However, the presence of lower energy bands in the emission spectra of the CdSe NCs synthesized at the shortest residence times is typical of smaller colloidal NCs, with recombination at intraband gap defect sites on the surface. ${ }^{1 a}$ 


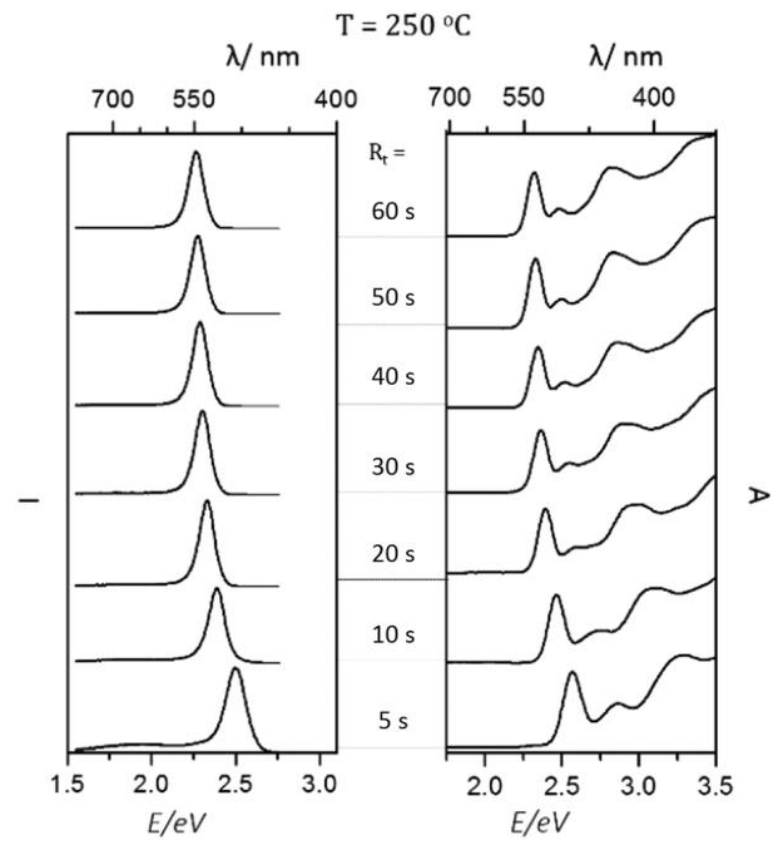

Figure 3 : Photoluminescence (excitation wavelength, $\lambda_{e x}=425 \mathrm{~nm}$ ) (left) and absorbance (right) spectra of CdSe NCs with residence times of $5,10,20,30,40,50$ and 60 s at $T=250^{\circ} \mathrm{C}$ and $P=10 \mathrm{MPa}$.

The quantitative photoluminescence properties of CdSe NCs synthesized in supercritical hexane are illustrated in Fig. 4. The FWHM of the PL peaks decreased with increasing residence time, reaching a constant value (Fig. $4 \mathrm{a}$, top panel), which is in good agreement with the expected narrowing of the RTD. ${ }^{14 a, 17}$

(a)

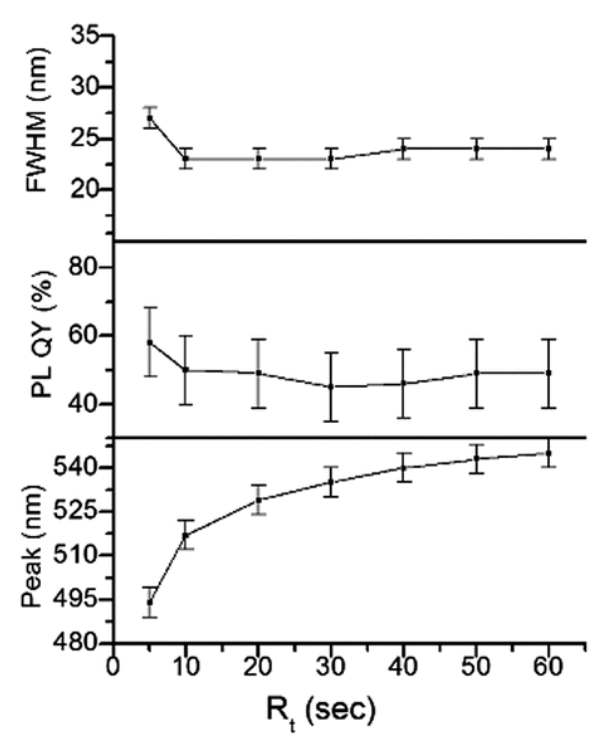

(b)

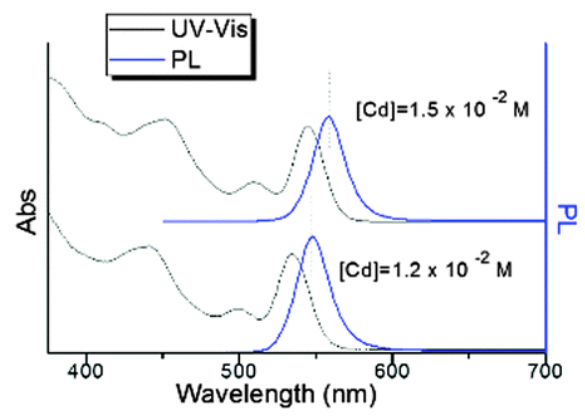

(c)

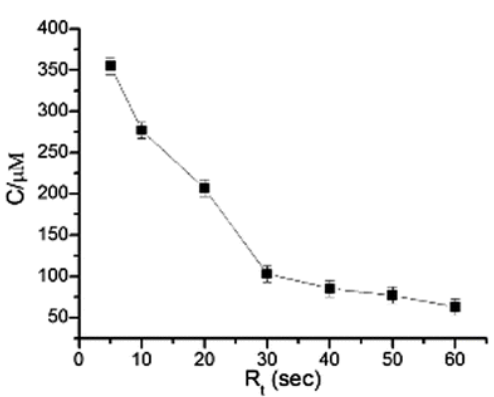

Figure 4 : (a) Photoluminescence characteristics of CdSe NCs synthesized at $250^{\circ} \mathrm{C}, 10 \mathrm{MPa}$ : FWHM (top panel), PL QY (middle panel, $\lambda_{\text {ex }}=425 \mathrm{~nm}$ ) and $\lambda_{\max }$ of emission (bottom panel); (b) absorption and photoluminescence spectra of NCs at two concentrations of $\mathrm{Cd}$ with same residence times $\left(R_{t}=60 \mathrm{~s}\right)$ at $250^{\circ} \mathrm{C}, 10 \mathrm{MPa}$; (c) concentration (C) of the CdSe NCs vs. $R_{t}$ at $250^{\circ} \mathrm{C}, 10 \mathrm{MPa}$.

However, the PL quantum yields of the as-prepared CdSe QDs were essentially constant regardless of residence time (45-50\%, Fig. 4a, middle panel). With the shortest residence time (5 s), the quantum yield was found to be significantly higher than the other NCs due to efficient capping of oleylamine over the smaller CdSe NCs (ca. $2.2 \mathrm{~nm}$ ). The high photoluminescence efficiency (45-58\%) of the CdSe QDs obtained with this approach demonstrates the capabilities of our strategy, since the controlled growth of the particles in presence of a suitable surface ligand (oleylamine) ensured less defects on the QDs surface, leading to high quantum efficiency 
and bright photoluminescence with pure colour. As an example, the calculated QY of the green-yellow emitting QDs $\left(\lambda_{\mathrm{em}}=549 \mathrm{~nm}, R_{\mathrm{t}}=60 \mathrm{~s}\right)$ was $77 \%$ at $\lambda_{\mathrm{ex}}=535 \mathrm{~nm}$ based on the ratio of intensity in the excitation spectrum to the absorbance. ${ }^{19}$ With the concentration of $\mathrm{Cd}$ precursor being $1.2 \times 10^{-2}(\mathrm{M})$, the reaction set up successfully yielded high quality CdSe NCs emitting in the green-yellow window (Fig. 4a, bottom panel). Increasing the concentration of the precursor to $1.5 \times 10^{-2} \mathrm{M}$ led to the red shift of photoluminescence of the CdSe QDs indicating larger size (Fig. 4b), as previously reported. ${ }^{14 a, 20}$ The concentration of NCs for the samples obtained in aliquots collected at different residence times was calculated using the absorption wavelength of the lowest energy transition (Fig. 4c) whose evolution was aforementioned discussed. ${ }^{2}$

Similar results were obtained when the reaction was run at $310^{\circ} \mathrm{C}$. The PL spectra showed sharp FWHM (24$27 \mathrm{~nm}$ ) for the short residence times $(5 \mathrm{~s}, 10 \mathrm{~s})$, and multiple excitonic features were observed in the absorption spectra, with the first and second excitonic peaks closer to each other as compared to the absorption spectra of ZB CdSe QDs synthesized at $250{ }^{\circ} \mathrm{C} .{ }^{18}$

These optical properties show that at this temperature a würtzite (W) crystal structure is obtained (Fig. 5). However, one can notice considerable broadening of the PL spectra and disappearance of the excitonic peaks in the absorption spectra with increasing residence times (20-60 s). We can assume that this might be caused by a secondary nucleation, accompanied by uncontrolled growth of smaller würtzite NCs inside the reactor.

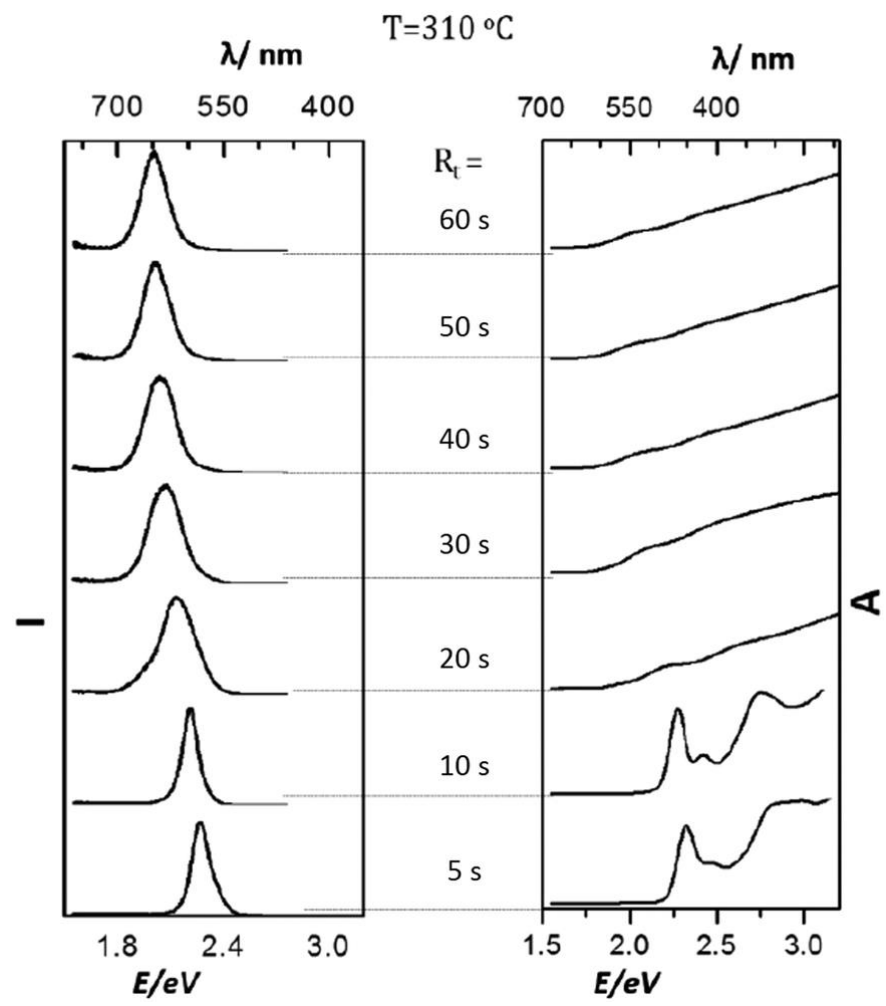

Figure 5 : Photoluminescence (left) and absorption (right) spectra of CdSe NCs with residence times 5, 10, 20, 30, 40, 50 and $60 \mathrm{~s}$ at $T=310^{\circ} \mathrm{C}$ and $P=10 \mathrm{MPa}\left(\lambda_{e x}=425 \mathrm{~nm}\right)$.

The PL characteristics of the CdSe NCs synthesized at $310^{\circ} \mathrm{C}$ were somewhat less impressive as compared to those synthesized at $250^{\circ} \mathrm{C}$. With very short residence times (5 and $10 \mathrm{~s}$ ), the FWHM was sharper (24-27 nm), but with increasing residence times, the FWHM became broader ( $>40 \mathrm{~nm}$ ) signifying the uncontrolled growth of the larger NCs (Fig. 6a, top panel). The photoluminescence quantum yield was also initially higher for the green emitting CdSe NCs ( 50\%), but then dropped considerably for the orange-red emitting QDs, potentially due to the improper surface passivation of the larger surface area of the orange-red emitting QDs (Fig. 6a, middle panel). These issues aside, we could obtain CdSe QDs emitting in a broader spectral region from green to red (550-625 nm, Fig. 6a, bottom panel) at $310^{\circ} \mathrm{C}$. It is also interesting to mention that by increasing the concentration of the $\mathrm{Cd}$ and Se precursors (2.4 and $3.6 \times 10^{-2} \mathrm{M}$, respectively), the broadness of the size distribution of the CdSe NCs can be considerably reduced (FWHM $=42 \mathrm{~nm}$, Fig. 6b). This is because by increasing 
the initial concentration of the monomer both the focusing time and the focused size are increased, leading to tighter size distribution of the NCs and reduction of the FWHM of the PL spectra. ${ }^{20}$

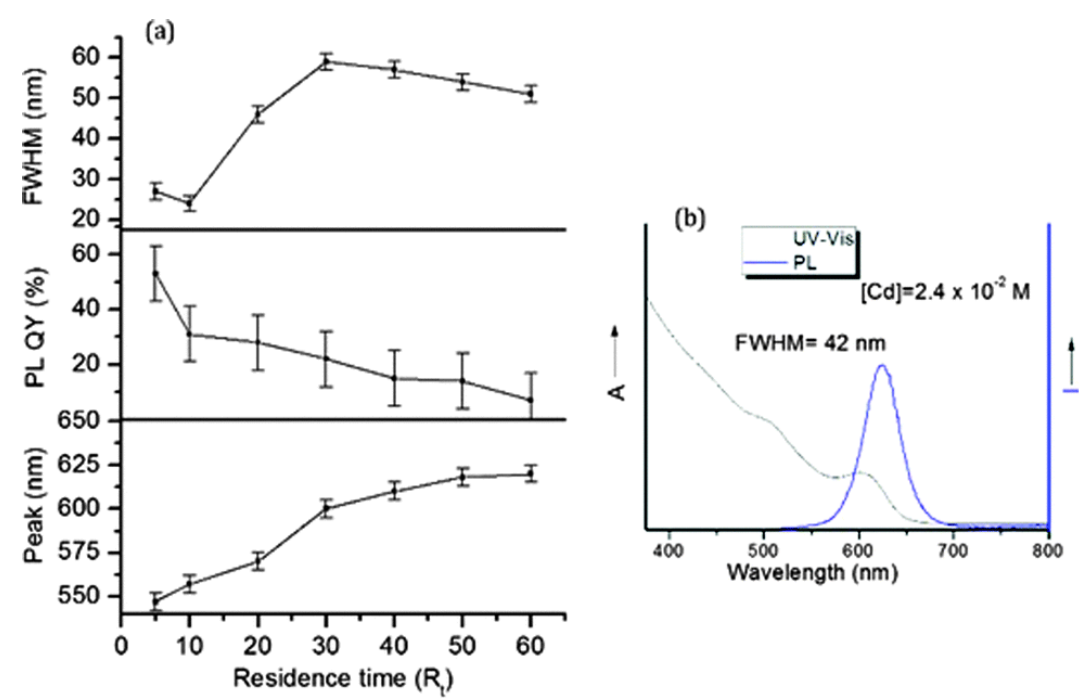

Figure 6 : (a) Photoluminescence characteristics of CdSe NCs synthesized at $310^{\circ} \mathrm{C}, 10 \mathrm{MPa}$ : FWHM (top panel), PL QY (middle panel, $\lambda_{e x}=425$ and $525 \mathrm{~nm}$ ) and $\lambda_{\max }$ of emission (bottom panel); (b) absorption and PL spectra of CdSe QDs at a concentration of $C d$ of $2.4 \times 10^{-2} \mathrm{M}$ with a 30 s residence time, $P=10 \mathrm{MPa}, \mathrm{T}=310^{\circ} \mathrm{C}$.

\section{Nanocrystal structure}

The preliminary structural results obtained from UV-vis absorption and PL were confirmed by powder-XRD measurements, showing that the CdSe NCs synthesized at $250{ }^{\circ} \mathrm{C}$ exhibit indeed a zinc blende (ZB) structure, while a würtzite (W) structure is obtained at $310^{\circ} \mathrm{C}$ (Fig. 7). The diffraction pattern of CdSe NCs synthesized at $310^{\circ} \mathrm{C}$ showed reflections of (102) and (103) planes at $2 \theta$ angles of 35.1 and $45.8^{\circ}$, respectively (Fig. $7 \mathrm{~d}$ ), in accordance with the Miller indices of W CdSe (JCPDS no. 08-0459). These reflections were absent (Fig. 7b) in case of CdSe NCs synthesized at $250{ }^{\circ} \mathrm{C}$, corresponding to ZB crystal structures (JCPDS no. 19-0191).
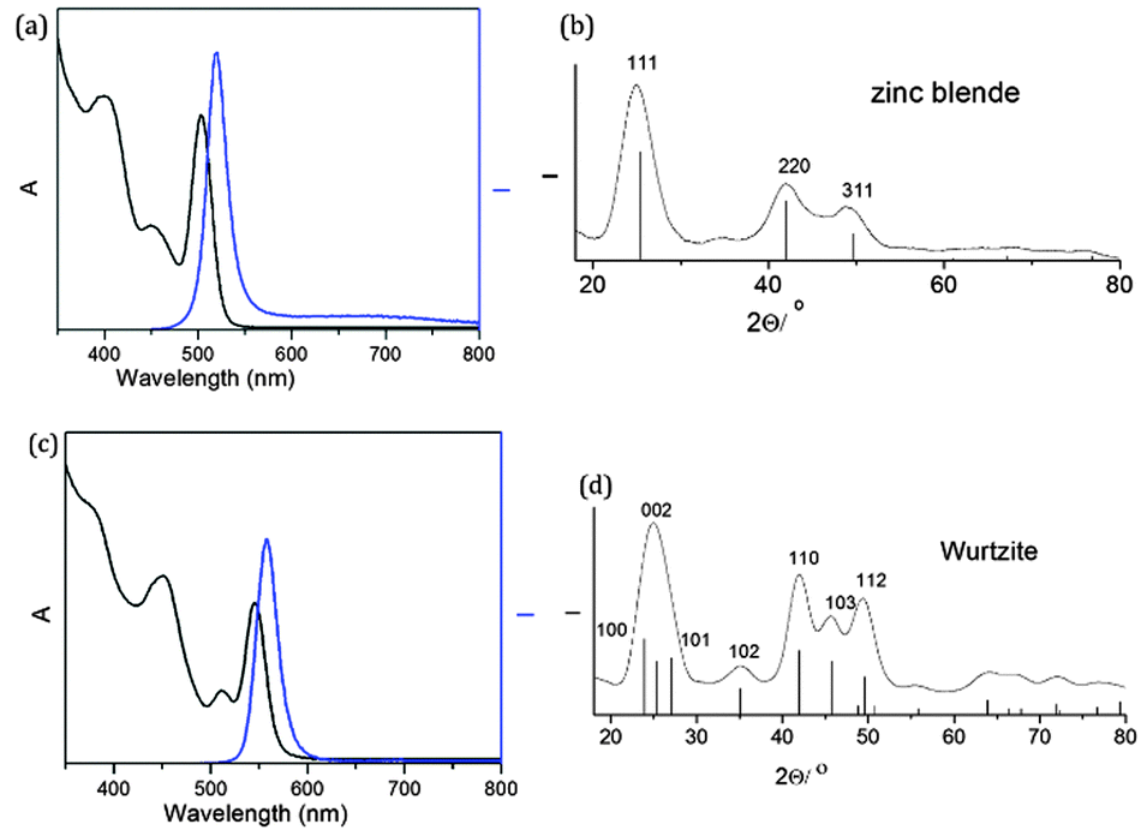

Figure : 7 Absorbance and $P L\left(\lambda_{e x}=425 \mathrm{~nm}\right)$ spectra of $C d$ Se quantum dots synthesized in supercritical hexane $(P=10$ $\mathrm{MPa}$ ) (a) at $250{ }^{\circ} \mathrm{C}, R_{t}=10 \mathrm{~s}$; and (c) at $310^{\circ} \mathrm{C}, R_{t}=10 \mathrm{~s}$; powder XRD patterns (b) at $250^{\circ} \mathrm{C}, R_{t}=30 \mathrm{~s}$, zinc blende and (d) $310^{\circ} \mathrm{C}, R_{t}=60 \mathrm{~s}$, würtzite 


\section{Transmission electron microscopy}

The size and particle size distribution were quantified by transmission electron microscopy. The results are shown in Fig. 8. The average diameter of the obtained particles was $2.4-2.9 \mathrm{~nm}$ for $R_{\mathrm{t}}$ ranging from (20-60 s) at $250{ }^{\circ} \mathrm{C}$ (Fig. 8a). However, the size distribution remains almost constant and monodisperse $(\sigma \leq 5 \%)$ in all cases (Fig. 8b), consistent with the excellent optical properties of these materials.
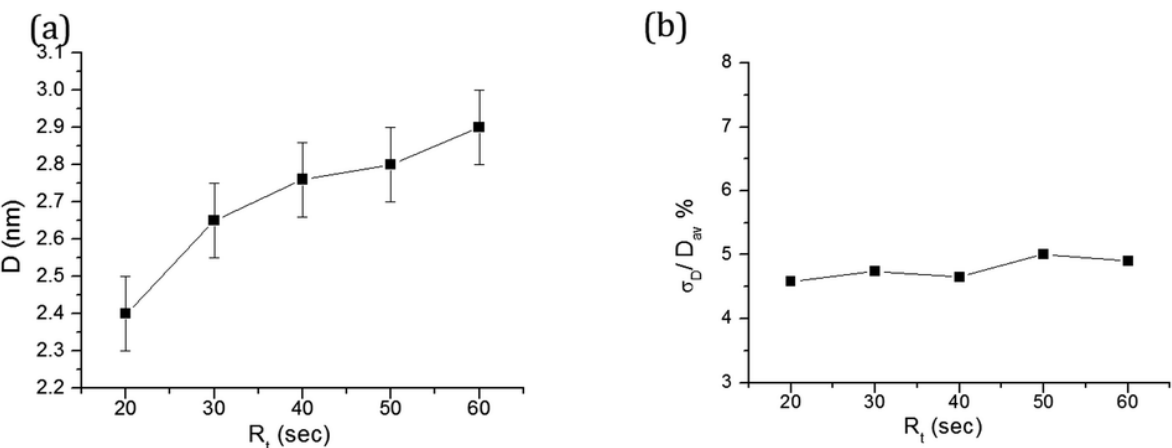

Figure 8 : (a) Diameter of NCs (nm) vs. $R_{t}$ for the CdSe QDs synthesized at $T=250^{\circ} \mathrm{C}, P=10 \mathrm{MPa}$ and (b) size distribution vs. $R_{t}$.

TEM images of CdSe NCs show uniformly sized NCs with narrow size distribution ( $\sigma \leq 5 \%)$. An aliquot collected with $R_{\mathrm{t}}=30 \mathrm{~s}$ at $250^{\circ} \mathrm{C}$ showed average particle diameter of $2.7 \mathrm{~nm}$ with narrow size distribution $(\sigma=4.7 \%$, Fig. 9a and b). As expected, high resolution TEM images show excellent crystallinity of the CdSe particles. The low size dispersity resulted in the formation of hexagonal superlattices on the TEM grids. The interplanar spacing was found to be $3.33 \AA$ for ZB CdSe QDs at $250^{\circ} \mathrm{C}$ with $R_{\mathrm{t}}=40 \mathrm{~s}$ (Fig. 9c and d).

The reactor likewise generated narrow size distributed particles at $310{ }^{\circ} \mathrm{C}$, with $\sigma=5 \%$ for short $R_{\mathrm{t}}(5$ and $10 \mathrm{~s})$, as exemplified in Fig. 9e and f. This low size dispersity indicates the fact that although the reaction is much faster at $310^{\circ} \mathrm{C}$, there is no evidence of secondary nucleation for short $R_{\mathrm{t}}$. In contrast, this phenomenon seemed to occur at $310^{\circ} \mathrm{C}$ for larger $R_{\mathrm{t}}(>10 \mathrm{~s})$, as previously observed in the broad PL peaks, leading to a broadening of size distribution (see ESI $+{ }^{20,22}$ HRTEM analysis was performed to confirm the crystallinity of the CdSe QDs synthesized at $310^{\circ} \mathrm{C}$. Single particle image showed the presence of lattice fringes with the interplanar lattice spacing found to be $3.54 \AA$ corresponding to the würtzite structure of CdSe NCs (Fig. 9g). 

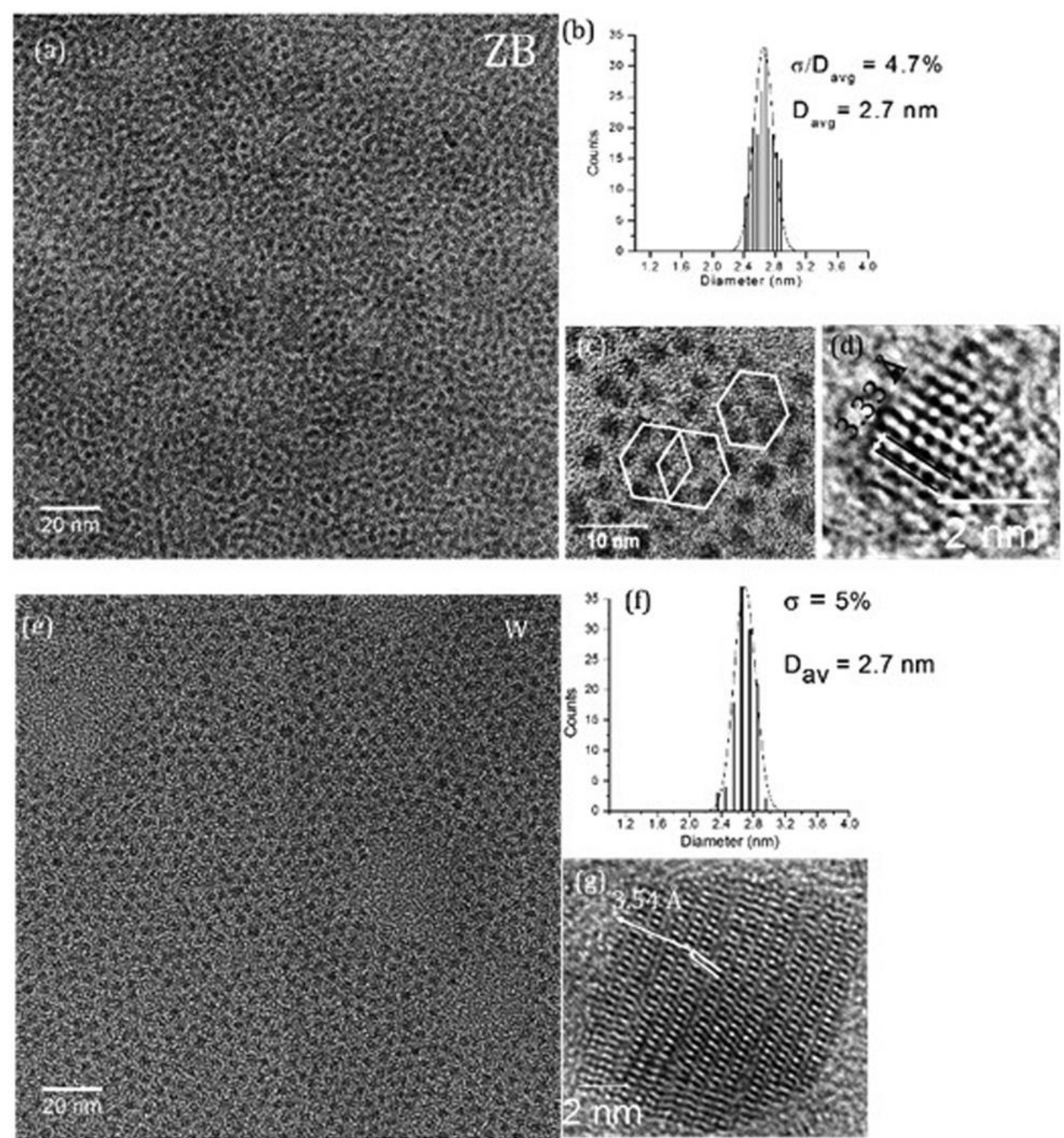

Figure : 9 (a) TEM image of ZB CdSe QDs synthesized at $250{ }^{\circ} \mathrm{C}, 10 \mathrm{MPa}, R_{t}=30 \mathrm{~s}$; (b) histogram of particle size distribution ( $\sigma=4.7 \%$ ) with average particle diameter $2.7 \mathrm{~nm}$, at $250{ }^{\circ} \mathrm{C}, 10 \mathrm{MPa}, R_{t}=30 \mathrm{~s}$; (c) and (d) HRTEM images of the ZB CdSe NCs showing hexagonal superlattices and lattice fringes with interplanar spacing of $3.33 \AA$ respectively (at $250{ }^{\circ} \mathrm{C}, 10 \mathrm{MPa}, R_{t}=40 \mathrm{~s}$ ); (e) TEM image of $\mathrm{W} C d S e N C s$ synthesized at $310^{\circ} \mathrm{C}, 10 \mathrm{MPa}$ and $R_{t}=10 \mathrm{~s}$; (f) histogram of size distribution of the $W$ CdSe NCs, at $310^{\circ} \mathrm{C}, 10 \mathrm{MPa}$ and $R_{t}=10 \mathrm{~s} ;(\mathrm{g})$ HRTEM images of the W CdSe synthesized at $T=$ $310^{\circ} \mathrm{C}, R_{t}=60 \mathrm{~s}$ showing lattice fringes with interplanar spacing of $3.54 \AA$.

\section{Production rate}

Large scale fabrication of high quality QDs is essential for realizing the promise of their application in the real world. Production issues from microfluidics nanomaterials synthesis approaches are currently investigated, ${ }^{23}$ demonstrating the real need for alternative methods. Indeed, continuous microscale synthesis of CdSe QDs is a potentially scalable technology; however, the small volumes and flowrates of "on chip" methods are not compatible with large scale production. In comparison, the present strategy for continuous synthesis of CdSe QDs in supercritical fluids is far more efficient for large scale production, producing up to $200 \mathrm{mg}$ of high quality CdSe QDs per hour, depending on the targeted size (see ESIt for more details).

Given that our approach can be performed in parallel, gram scale production (per hour) can be easily achieved in the laboratory, with industrial scale possible with an appropriate reactor design. This scalability coupled with the use of inexpensive solvent and precursors makes our strategy particularly attractive for large-scale production of QDs. 


\section{Conclusion}

In conclusion, we have developed a scalable technology using supercritical fluids to prepare high quality zinc blende (ZB) and würtzite (W) CdSe QDs with narrow size distribution ( $\sigma \leq 5 \%)$, high photoluminescence quantum yields $(45<\mathrm{QY}<55 \%)$ and well-defined crystallinity. The coupling of supercritical fluid technology with continuous microscale processing creates a less expensive and more efficient system for synthesizing CdSe QDs with high reproducibility. This approach overcomes the common obstacles encountered in the batch mode synthesis, e.g. expensive solvents, high viscosity and low diffusivity. The good control of the crystal phase (ZB or $W$ ) provides means for future orientated growth in overcoating steps (for instance by ZnS or CdS). Additionally, the continuous synthesis approach provides a high production rate (up to $200 \mathrm{mg}$ per hour), thus reducing the production cost and providing a step towards large-scale production of CdSe QDs. This strategy could also be applied to other types of nanomaterials synthesis.

Acknowledgements : The project was financially supported by CEFIPRA (project 4805-1), the CNRS, the French Ministry of Education and Research and Région Aquitaine. We thank Ms S. Buffière, PLACAMAT for recording TEM images.

\section{Notes and references}

1. (a) A. M. Smith and S. Nie, Acc. Chem. Res., 2010, 43, 190 ; (b) D. J. Norris and M. G. Bawendi, Phys. Rev. B: Condens. Matter Mater. Phys., 1996, 53(24), 16338 ; (c) L. E. Brus, J. Chem. Phys., 1984, 80, 4403.

2. (a) P. Kambhampati, J. Phys. Chem. Lett., 2012, 3, 1182 ; (b) S. Coe, W.-K. Woo, M. Bawendi and V. Bulović, Nature, 2002, 420, 800 ; (c) V. I. Klimov, S. A. Ivanov, J. Nanda, M. Achermann, I. Bezel, J. McGuire and A. Piryatinski, Nature, 2007, 447, 441.

3. (a) I. Gur, N. A. Fromer, M. L. Geier and A. P. Alivisatos, Science, $2005,310,462$; (b) W. U. Huynh, J. J. Dittmer and A. P. Alivisatos, Science, 2002, 295, 2425 ; (c) P. V. Kamat, J. Phys. Chem. C, 2008, 112, 18737.

4. (a) S. J. Rosenthal, J. C. Chang, O. Kovtun, J. R. McBride and I. D. Tomlinson, Chem. Biol., 2011, 18, 10 ; (b) P. Alivisatos, Nat. Biotechnol., 2004, 22, 47 ; (c) W. C. W. Chan and S. Nie, Science, 1998, 281, 2016.

5. B. S. Mashford, M. Stevenson, Z. Popovic, C. Hamilton, Z. Zhou, C. Breen, J. Steckel, V. Bulovic, M. Bawendi, S. Coe-Sullivan and P. T. Kazlas, Nat. Photonics, 2013, 7, 407.

6. (a) X. Dai, Z. Zhang, Y. Jin, Y. Niu, H. Cao, X. Liang, L. Chen, J. Wang and X. Peng, Nature, 2014, 515, 96 ; (b) S. Kim, S. H. Im and S.-W. Kim, Nanoscale, 2013, 5, 5205.

7. (a) C. B. Murray, D. J. Norris and M. G. Bawendi, J. Am. Chem. Soc., 1993, 115, 8706 ; (b) X. Peng, L. Manna, W. Yang, J. Wickham, E. Scher, A. Kadavanich and A. P. Alivisatos, Nature, 2000, 404, 59.

8. H. Nakamura, Y. Yamaguchi, M. Miyazaki, H. Maeda, M. Uehara and P. Mulvaney, Chem. Commun., 2002, 2844.

9. B. K. H. Yen, A. Günther, M. A. Schmidt, K. F. Jensen and M. G. Bawendi, Angew. Chem., Int. Ed., 2005, $44,5447$.

10. E. M. Chan, A. P. Alivisatos and R. A. Mathies, J. Am. Chem. Soc., 2005, 127, 13854. 
11. T. Gervais and K. F. Jensen, Chem. Eng. Sci., 2006, 61, 1102.

12. (a) K. F. Jensen, Chem. Eng. Sci., 2001, 56, 293 ; (b) S. Marre and K. F. Jensen, Chem. Soc. Rev., 2010, $39,1183$.

13. C. A. Eckert, B. L. Knutson and P. G. Debenedetti, Nature, 1996, 383, 313.

14. (a) S. Marre, J. Park, J. Rempel, J. Guan, M. G. Bawendi and K. F. Jensen, Adv. Mater., 2008, 20, 4830 ;

(b) J. Baek, P. M. Allen, M. G. Bawendi and K. F. Jensen, Angew. Chem., Int. Ed., 2011, 50, 627.

15. A. Chakrabarty, S. Chatterjee and U. Maitra, J. Mater. Chem. C, 2013, 1, 2136.

16. M. Grabolle, M. Spieles, V. Lesnyak, N. Gaponik, A. Eychmüller and U. Resch-Genger, Anal. Chem., $2009,81,6285$.

17. S. Marre, Y. Roig and C. Aymonier, J. Supercrit. Fluids, 2012, 66, 251.

18. O. Chen, X. Chen, Y. Yang, J. Lynch, H. Wu, J. Zhuang and Y. C. Cao, Angew. Chem., Int. Ed., 2008, 47, 8638.

19. The quantum yield values were further calculated at different excitation wavelengths by taking into consideration the excitation and emission spectra of the green-yellow emitting QDs $\left(\lambda_{\mathrm{em}}=549 \mathrm{~nm}, R_{\mathrm{t}}=\right.$ $60 \mathrm{~s})$. By plotting the ratio of PL excitation intensity to absorbance $(I / A)$ against wavelength of excitation, we could correlate it with the QY values taking into consideration the experimentally determined QY of $49 \%$ at $\lambda_{\mathrm{ex}}=425 \mathrm{~nm}$. Thus, the maximum QY value at $\lambda_{\mathrm{ex}}=535 \mathrm{~nm}$ was $77 \%$ (see ESI,_ Fig. S1-S4).

20. X. Peng, J. Wickham and A. P. Alivisatos, J. Am. Chem. Soc., 1998, 120, 5343.

21. W. W. Yu, L. Qu, W. Guo and X. Peng, Chem. Mater., 2003, 15, 2854.

22. C. B. Murray, C. R. Kagan and M. G. Bawendi, Annu. Rev. Mater. Sci., 2000, 30, 545.

23. (a) V. Sebastian, M. Arruebo and J. Santamaria, Small, 2014, 10, 835 ; (b) L. Gomez, V. Sebastian, S. Irusta, A. Ibarra, M. Arruebo and J. Santamaria, Lab Chip, 2014, 14, 325. 